\title{
The Effects of Biofilm Care on Subgingival Bacterial Motility and Halitosis
}

\author{
Yu-Rin $\mathrm{Kim}^{\dagger}$ \\ Department of Dental Hygiene, Silla University, Busan 46958, Korea
}

\begin{abstract}
Background: Oral diseases are caused by various systemic and local factors, the most closely related being the biofilm. However, the challenges involved in removing an established biofilm necessitate professional care for its removal. This study aimed to evaluate and compare the effects of professional self and professional biofilm care in healthy patients to prevent the development of periodontal diseases.

Methods: Thirty-seven patients who visited the dental clinic between September 2018 and February 2019 were included in this study. Self-biofilm care was performed by routine tooth brushing and professional biofilm care was provided using the toothpick method (TPM) or the oral prophylaxis (OP) method using a rubber cup. Subgingival bacterial motility and halitosis (levels of hydrogen sulfide, $\mathrm{H}_{2} \mathrm{~S}$; methyl mercaptan, $\mathrm{CH}_{3} \mathrm{SH}$; and di-methyl sulfide, $\left(\mathrm{CH}_{3}\right)_{2} \mathrm{~S}$ ) were measured before, immediately after, and 5 hours after the preventive treatment in the three groups. Repeated measures analysis of variance test was performed to determine significant differences among the groups.

Results: TPM was effective immediately after the prevention treatment, whereas OP was more effective after 5 hours (proximal surfaces, $F=16.353$, $p<0.001$; smooth surfaces, $F=66.575, p<0.001$ ). The three components responsible for halitosis were effectively reduced by professional biofilm care immediately after the preventive treatment; however, self-biofilm care was more effective after 5 hours $\left(\mathrm{H}_{2} \mathrm{~S}, \mathrm{~F}=3.564, \mathrm{p}=0.011 ; \mathrm{CH}_{3} \mathrm{SH}\right.$, $\left.F=6.657, p<0.001 ;\left(\mathrm{CH}_{3}\right)_{2} \mathrm{~S}, \mathrm{~F}=21.135, \mathrm{p}<0.001\right)$.

Conclusion: To prevent oral diseases, it is critical to monitor the biofilm. The dental hygienist should check the oral hygiene status and the ability of the patient to administer oral care. Professional biofilm care should be provided by assessing and treating each surface of the tooth. We hope to strengthen our professional in biofilm care through continuous clinical research.
\end{abstract}

Key Words: Bacteria, Dental care, Halitosis, Oral health

\section{Introduction}

Currently, gingivitis and periodontal disease are the second most common diseases encountered during outpatient care, while two of the tenth most common diseases seen are dental caries and diseases of the periradicular tissues; furthermore, health care costs have increased to $12.7 \%$ than the previous year ${ }^{1)}$. Oral diseases are mostly caused due to the destruction of the teeth and the alveolar bone resulting in tooth loss; hence, the prevention of damage to the tooth and the adjacent structures is of utmost importance ${ }^{2)}$. Oral disease is caused by various systemic and local factors, but the most closely related factor is the dental biofilm ${ }^{3)}$. Among the 500 species of bacteria present in the dental biofilm, those associated with periodontal disease (such as Actinobacillus actinomycetemcomitans and Porphyromonas gingivalis) are located subgingivally ${ }^{4)}$. Alternatively, Fusobacterium nucleatum is mostly detected in the gingival sulcus and plays a key role in coaggregation with other bacterial species ${ }^{5)}$. Bacteria such as $P$. gingivalis, Treponema denticola, Tannerella forsythia, and Prevotella intermedia are reported to cause foul smells in the oral cavity and have a major influence on halitosis ${ }^{6)}$. Patients with a periodontal disease generally complain of halitosis, caused by the bacteria living in the periodontal pocket and the lingual mucosa by producing volatile sulfur compounds (VSCs) $^{7)}$. P. gingivalis, T. denticola, and T. forsythia 
produce trypsin-like enzymes that hydrolyze the synthetic peptide N-benzoyl-DL-arginine-2-naphthylamide. These trypsin-like enzymes produce VSCs by hydrolyzing the sulfur-containing amino acids cysteine and methionine ${ }^{8)}$. VSCs consist of hydrogen sulfide $\left(\mathrm{H}_{2} \mathrm{~S}\right)$, methyl mercaptan $\left(\mathrm{CH}_{3} \mathrm{SH}\right)$, and dimethyl sulfide $\left(\left[\mathrm{CH}_{3}\right]_{2} \mathrm{~S}\right)$, which can be accurately and objectively measured using a portable gas chromatograph such as Oral $\mathrm{Chroma}^{\mathrm{TM}}$ (Model no. CHM-1; Abilit, Osaka, Japan) ${ }^{9}$. Hence, the provision of systematic and effective care using these types of equipment is essential for the prevention of halitosis. An increase in the proportion and motility of the subgingival bacteria will result in increased amounts of bacterial toxins and metabolic products. This will not only cause periodontal disease by destroying the periodontal tissue and irritating the immune system ${ }^{10)}$, but also have a negative effect on halitosis. Therefore, it is important to monitor and control the bacterial population in the subgingival biofilm.

Tooth brushing is the most basic and essential method of dental biofilm care; yet, additional professional care is required because most adults do not demonstrate adequate tooth brushing habitsprofessional ${ }^{11}$. Professional biofilm care methods include professional mechanical tooth cleaning (PMTC) and the toothpick method (TPM). For the PMTC, rubber cups are more commonly used instead of EVA-tips in clinical practice, whereas for the TPM, a 2-row toothbrush is used ${ }^{12}$. Professional biofilm care is known to be useful for the treatment of proximal tooth surfaces and the subgingival region thereby effectively eliminating halitosis ${ }^{13,14)}$. The massaging effect of the TPM aids in the treatment of the proximal surfaces, which are the first sites of inflammation; thus, this method enables the treatment of areas that are unaffected by normal tooth brushing alone ${ }^{15)}$. Recently, removal of dental calculus has been added to the national insurance coverage, leading to an increase in the number of patients in the dental clinic. Partial application of oral prophylaxis (OP) is recommended after calculus removal owing to enamel damage, and this needs to be performed efficiently and precisely on each of the dental surfaces ${ }^{16}$.

In this study, the effects of tooth brushing (representing self-biofilm care) and TPM or OP using rubber cups (representing professional biofilm care) on the different surfaces of the teeth were examined. Additionally, to examine the continuity of the prophylactic treatment, we analyzed the subgingival bacterial motility and halitosis over time with the aim of collecting data to support the provision of additional, systematic and specialized oral care.

\section{Materials and Methods}

\section{Participants}

Thirty-seven patients who visited the prophylactic treatment room at the dental clinic in Busan between September 2018 and February 2019 were included in this study. The patients were aged between 20 and 29 years, presented with a healthy oral cavity, and voluntarily consented to participate in the study. The required sample size was 16 individuals, calculated using $\mathrm{G}^{*}$ power 3.1.9.2 (statistical power analyses for Windows and Mac) software. The exclusion criteria for the study were as follows: the presence of a prosthesis or restoration that could affect the results; use of an orthodontic device; consumption of medications; and habits such as smoking or alcohol consumption. The experimental groups consisted of 13 patients who received OP and 12 patients who received TPM. The remaining 12 patients in the control group performed general tooth brushing.

\section{Research methods}

\section{1) Research flowchart}

Subgingival bacterial motility and halitosis were measured initially in each group before and immediately after the respective prophylactic treatments. Bacteria attach to lectins with a glycoprotein structure, in the acquired pellicle or the by-products of food within two hours ${ }^{17)}$. Therefore, in order to check the continuity of the prophylactic treatment, both bacterial motility and halitosis were measured a third time after 5 hours. After the 2nd measurement and before the 3rd measurement, participants were required to drink one cup of water. To minimize the differences in measurements, the entire procedure was conducted by one dental hygienist and one 
board-certified preventive dentist, both having at least 10 years of experience in their respective fields (Fig. 1).

\section{2) Factors affecting periodontal disease}

\section{(1) Motility of subgingival bacteria}

All teeth were categorized based on surfaces, such as, into the proximal surfaces (mesial and distal), occlusal surface, buccal surface, and lingual surface. A sterilized \#11 or \#12 explorer was inserted 1 2 mm subgingivally and moved back and forth twice to collect the bacteria. For the proximal surfaces, the mesial and distal surfaces were combined, and for the smooth surfaces, the buccal and lingual surfaces were combined. In order to assess the subgingival bacterial motility, a video recording of at least 10 seconds duration was obtained using a microscope (BA310; Motic, Hong Kong, China) at $400 \times$ magnification. The recorded image was divided into quarters, and the movements per second were analyzed. To improve the objectivity of the analysis, a board-certified preventive dentist and a dental hygienist specialized in prevention compiled the assessment criteria and individually assessed the videos. In the event of a severe error, the video was re-assessed and the results were adjusted. The investi-

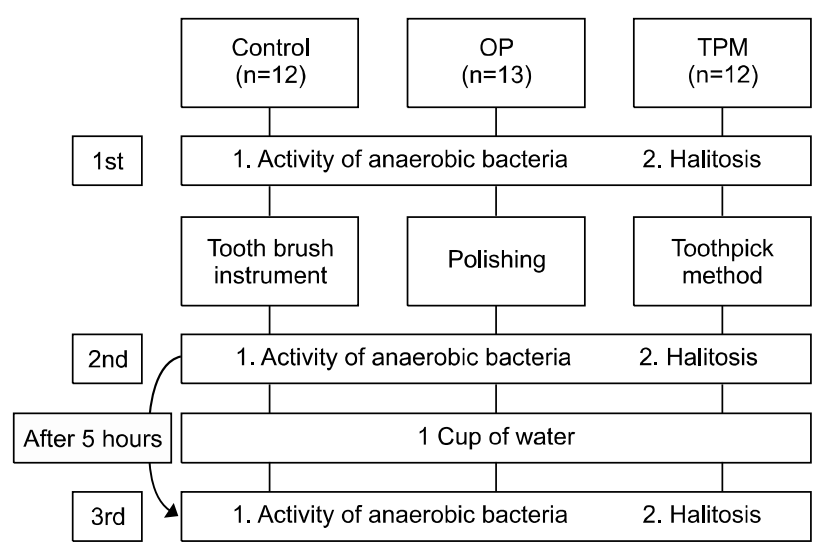

Fig. 1. Study method. OP: oral prophylaxis, TPM: toothpick method. gators showed $96 \%$ agreement on the assessment findings.

\section{(2) Halitosis}

Halitosis was measured using an Oral Chroma device (Model no. CHM-1). First, the participants were instructed to close their mouths for at least 3 minutes. Subsequently, a sample was collected using a specialized syringe and measurements were taken for 8 minutes. The relative amounts of $\mathrm{H}_{2} \mathrm{~S}, \mathrm{CH}_{3} \mathrm{SH}$, and $\left(\mathrm{CH}_{3}\right)_{2} \mathrm{~S}$ were analyzed separately; hence, compounds with a thiol moiety, containing sulfur, were accurately measured and analyzed. For the assessment of halitosis, the measurement value of each component was marked as ' + ', if it exceeded the threshold value for halitosis and '- ', if it was lower than the threshold value. Thus four types of halitosis were assessed $(\text { Table } 1)^{18)}$.

\section{3) Data analysis}

The collected data were analyzed using the statistical analysis software IBM SPSS ver. 21.0 (IBM Corp., Armonk, NY, USA). A repeated measures ANOVA test was performed to analyze subgingival bacterial motility and halitosis by tooth surface and time point. To analyze the within-subject effects, multivariate tests were performed if the assumption of sphere city was not satisfied based on Mauchly's test of sphere city. Alternatively, tests of within-subject effects were performed if the assumption of sphere city was satisfied. Between-subject effects were analyzed by performing the tests of between-subject effects. Statistical significance was determined using a significance level of 0.05 .

\section{Results}

1. Changes in subgingival bacterial motility over time in the three groups

Subgingival bacterial activities on the proximal surfaces

Table 1. Method of Judging Halitosis ${ }^{18}$

\begin{tabular}{|c|c|c|c|c|}
\hline Pattern & Judgment sentence & $\mathrm{H}_{2} \mathrm{~S}$ & $\mathrm{CH}_{3} \mathrm{SH}$ & $\left(\mathrm{CH}_{3}\right)_{2} \mathrm{~S}$ \\
\hline I & The range, which hardly senses a smell, although there is halitosis & - & - & - \\
\hline II & The range, which senses halitosis a little & + & - & - \\
\hline III & The range, which periodontitis is suspected & + & + & + or - \\
\hline IV & Halitosis is suspected as the cause of food metabolism or medication & - & - & + \\
\hline
\end{tabular}


were as follows: before prophylactic treatment, the motility score was $18.83,18.92$, and 18.33 in the general tooth brushing, OP and TPM groups respectively; immediately after prophylactic treatment, motility the scores were 28.58, 11.92, and 10.42, respectively. Thus, the TPM group demonstrated the lowest scores. After 5 hours, motility scores were $50.25,18.08$, and 21.50 in the tooth brushing, OP, and TPM groups, respectively with lowest scores in the OP group. The differences among the three groups were significant $(\mathrm{F}=11.541, \mathrm{p}<0.001)$ and significant changes were noted over time $(\mathrm{F}=49.834, \mathrm{p}<$ 0.001 ). Additionally, the changes in bacterial motility over time on the proximal surfaces were significantly different among the three groups $(\mathrm{F}=16.353, \mathrm{p}<0.001)$. The subgingival bacterial activities on the smooth surfaces were as follows: before treatment, the bacterial motility scores were 68.92 in both, the general tooth brushing and OP groups, and 69.42 in the TPM group; immediately after prophylactic treatment, the motility scores were $36.42,36.42$, and 12.67, respectively, with the TPM group presenting with the lowest scores. After 5 hours, the motility scores were $68.92,68.92$, and 22.50 , respectively with lowest scores in the TPM group. The differences among the three groups were significant $(F=273.311, p<$ 0.001 ) and significant changes were observed over time $(\mathrm{F}=649.014, \mathrm{p}<0.001)$. The changes in bacterial motility over time on the smooth surfaces differed significantly among the three groups $(\mathrm{F}=66.575, \mathrm{p}<0.001$; Table 2, Fig. 2).

\section{Changes in halitosis components over time in the three groups}

The scores for changes in $\mathrm{H}_{2} \mathrm{~S}$ before prophylactic treatment were $1.58,1.54$ and 1.42 in the tooth brushing, OP, and TPM groups, respectively; immediately after treatment, the respective scores were $4.92,3.15$, and 1.83

Table 2. Activity of Subgingival Bacteria according to Groups Mean \pm standard Deviation

\begin{tabular}{clccclcr}
\hline Group $^{\mathrm{a}}$ & \multicolumn{1}{c}{ Part } & Baseline & After treat & After 5 hours & Source & F & p-value \\
\hline Control $(\mathrm{n}=12)$ & Interdental & $18.83 \pm 1.47$ & $28.58 \pm 2.84$ & $50.25 \pm 8.49$ & Group $^{\mathrm{b}}$ & 11.541 & $<0.001$ \\
& Smooth & $68.92 \pm 3.85$ & $36.42 \pm 6.67$ & $68.92 \pm 5.76$ & Time $^{\mathrm{b}}$ & 49.834 & $<0.001$ \\
OP $(\mathrm{n}=13)$ & Interdental & $18.92 \pm 3.48$ & $11.92 \pm 3.55$ & $18.08 \pm 4.48$ & Group*time $^{\text {b }}$ & 16.353 & $<0.001$ \\
& Smooth & $68.92 \pm 3.85$ & $36.42 \pm 6.67$ & $68.92 \pm 5.76$ & Group $^{\text {b }}$ & 273.311 & $<0.001$ \\
TPM $(\mathrm{n}=12)$ & Interdental & $18.33 \pm 3.63$ & $10.42 \pm 2.35$ & $21.50 \pm 8.84$ & Time $^{\mathrm{b}}$ & 649.014 & $<0.001$ \\
& Smooth & $69.42 \pm 4.94$ & $12.67 \pm 4.23$ & $22.50 \pm 4.54$ & Group*time $^{\text {b }}$ & 66.575 & $<0.001$ \\
\hline
\end{tabular}

p-values by repeated measures ANOVA test.

OP: oral prophylaxis, TPM: toothpick method.

${ }^{\mathrm{a}}$ Control: tooth brush instrument. ${ }^{\mathrm{b}}$ Interdental surface. ${ }^{\mathrm{c}}$ Smooth surface.
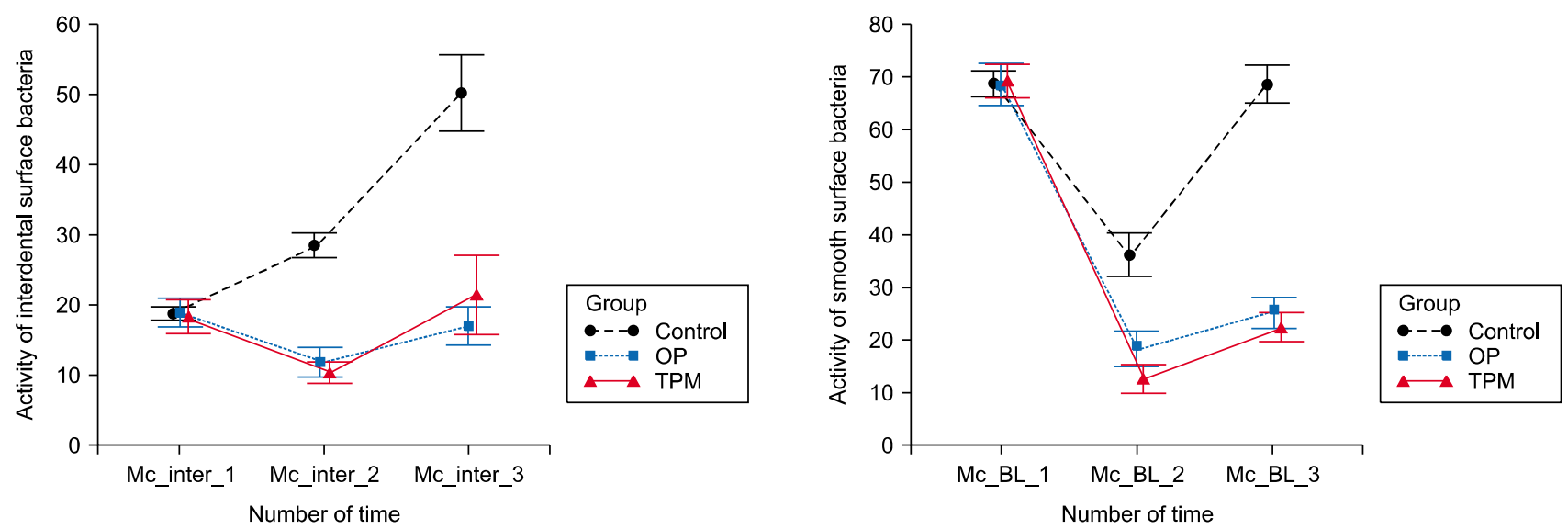

Fig. 2. Activity of subgingival bacteria according to groups. OP: oral prophylaxis, TPM: toothpick method. 
with lowest scores in the TPM group and after 5 hours, the scores were, respectively, $0.92,0.85$, and 1.08 , indicating lowest scores in the OP group. The differences among the three groups were significant $(\mathrm{F}=5.222, \mathrm{p}=0.011)$ and significant changes over time were noted $(\mathrm{F}=29.462, \mathrm{p}<$ 0.001). The changes in $\mathrm{H}_{2} \mathrm{~S}$ over time differed significantly among the three groups $(\mathrm{F}=3.564, \mathrm{p}=0.011)$. When we analyzed changes in $\mathrm{CH}_{3} \mathrm{SH}$, before prophylactic treatment, scores were 1.50, 1.62, and 1.67 in the general tooth brushing, OP, and the TPM groups, respectively; immediately after treatment, the scores were $6.75,2.38$, and 3.58, respectively, with the lowest in the OP group. After 5 hours, the scores were 1.50, 3.08, and 2.75 in the tooth brushing, OP, and TPM groups, respectively with lowest score in the general tooth brushing group. The differences among the three groups were not significant $(\mathrm{F}=2.542, \mathrm{p}=0.094)$; however, significant changes over time were noted $(\mathrm{F}=25.498, \mathrm{p}<0.001)$. Additionally, changes in $\mathrm{CH}_{3} \mathrm{SH}$ over time differed significantly among the three groups $(\mathrm{F}=6.657, \mathrm{p}<0.001)$. When we analyzed changes in $\left(\mathrm{CH}_{3}\right)_{2} \mathrm{~S}$, the scores before prophylactic treatment were $2.00,1.92$, and 1.50 in the general tooth brushing, OP, and TPM group respectively; immediately after treatment, the scores were $11.58,0.62$, and 0.75 , respectively with the lowest in the OP group. After 5 hours, the scores were $1.25,16.00$, and 10.08 in the tooth brushing, OP, and TPM groups, respectively and the general tooth brushing group exhibited the lowest score). The differences among the three groups were significant
$(\mathrm{F}=4.870, \mathrm{p}=0.014)$ and significant changes over time were observed $(\mathrm{F}=101.021, \mathrm{p}<0.001)$. Furthermore, the changes in $\left(\mathrm{CH}_{3}\right)_{2} \mathrm{~S}$ over time significantly differed among the three groups $(\mathrm{F}=21.135, \mathrm{p}<0.001$; Table 3, Fig. 3).

\section{Discussion}

Only a minority of the bacteria in the subgingival biofilm cause oral diseases in healthy individuals; the incidence of oral disease increases when the ratios of these subgingival bacteria increase ${ }^{4}$. Therefore, in order to maintain a healthy oral cavity, it is essential to eliminate the subgingival biofilm and provide professional oral care to prevent reattachment. Accordingly, in this study, we analyzed the dental surfaces separately to compare the effects of general tooth brushing, which represents selfbiofilm care, and TPM or OP using rubber cups, which represent professional biofilm care.

We examined changes in subgingival bacterial motility on the proximal surfaces of the teeth. Immediately after treatment, the motility score was increased by 9.75 in the patients who used the self-biofilm care method, but decreased by 7 and 7.42 in the OP and TPM groups indicating that TPM was the most effective immediately after treatment. To examine the continuity of the effect, subgingival bacterial motility was re-measured motility after 5 hours. Increased motility scores of 21.67 in the general tooth brushing group, 6.16 in the OP group, and 11.08 in the TPM group were observed, suggesting a more

Table 3. Halitosis according to Groups Mean \pm standard Deviation

\begin{tabular}{cllrrlrr}
\hline \multicolumn{1}{c}{ Group $^{\mathrm{a}}$} & Ingredient & Baseline & After treat & After 5 hours & Source & $\mathrm{F}$ & p-value \\
\hline Control $(\mathrm{n}=12)$ & $\mathrm{H}_{2} \mathrm{~S}$ & $1.58 \pm 1.44$ & $4.92 \pm 2.11$ & $0.92 \pm 0.99$ & Group $^{\mathrm{b}}$ & 5.222 & 0.011 \\
& $\mathrm{CH}_{3} \mathrm{SH}$ & $1.50 \pm 1.24$ & $6.75 \pm 3.12$ & $1.50 \pm 1.09$ & Time $^{\mathrm{b}}$ & 29.462 & $<0.001$ \\
& $\left(\mathrm{CH}_{3}\right)_{2} \mathrm{~S}$ & $2.00 \pm 1.95$ & $11.58 \pm 2.75$ & $1.25 \pm 1.06$ & Group*time $^{\mathrm{b}}$ & 3.564 & 0.011 \\
$\mathrm{OP}(\mathrm{n}=13)$ & $\mathrm{H}_{2} \mathrm{~S}$ & $1.54 \pm 1.39$ & $3.15 \pm 1.73$ & $0.85 \pm 1.07$ & Group $^{\mathrm{b}}$ & 2.542 & 0.094 \\
& $\mathrm{CH}_{3} \mathrm{SH}$ & $1.62 \pm 1.26$ & $2.38 \pm 1.19$ & $3.08 \pm 0.76$ & Time $^{\mathrm{b}}$ & 25.498 & $<0.001$ \\
& $\left(\mathrm{CH}_{3}\right)_{2} \mathrm{~S}$ & $1.92 \pm 1.66$ & $0.62 \pm 0.96$ & $16.00 \pm 4.08$ & Group $^{*}$ time $^{\mathrm{b}}$ & 6.657 & $<0.001$ \\
$\mathrm{TPM}(\mathrm{n}=12)$ & $\mathrm{H}_{2} \mathrm{~S}$ & $1.42 \pm 1.31$ & $1.83 \pm 1.03$ & $1.08 \pm 0.52$ & Group $^{\mathrm{b}}$ & 4.870 & 0.014 \\
& $\mathrm{CH}_{3} \mathrm{SH}$ & $1.67 \pm 1.16$ & $3.58 \pm 1.78$ & $2.75 \pm 1.22$ & Time $^{\mathrm{b}}$ & 101.021 & $<0.001$ \\
& $\left(\mathrm{CH}_{3}\right)_{2} \mathrm{~S}$ & $1.50 \pm 1.62$ & $0.75 \pm 0.87$ & $10.08 \pm 4.52$ & Group$^{*}$ time & 21.135 & $<0.001$ \\
\hline
\end{tabular}

p-values by repeated measures ANOVA test.

OP: oral prophylaxis, TPM: toothpick method.

${ }^{\text {a }}$ Control: tooth brush instrument. ${ }^{b} \mathrm{H}_{2} \mathrm{~S},{ }^{\mathrm{c}} \mathrm{CH}_{3} \mathrm{SH},{ }^{\mathrm{d}}\left(\mathrm{CH}_{3}\right)_{2} \mathrm{~S}$. 

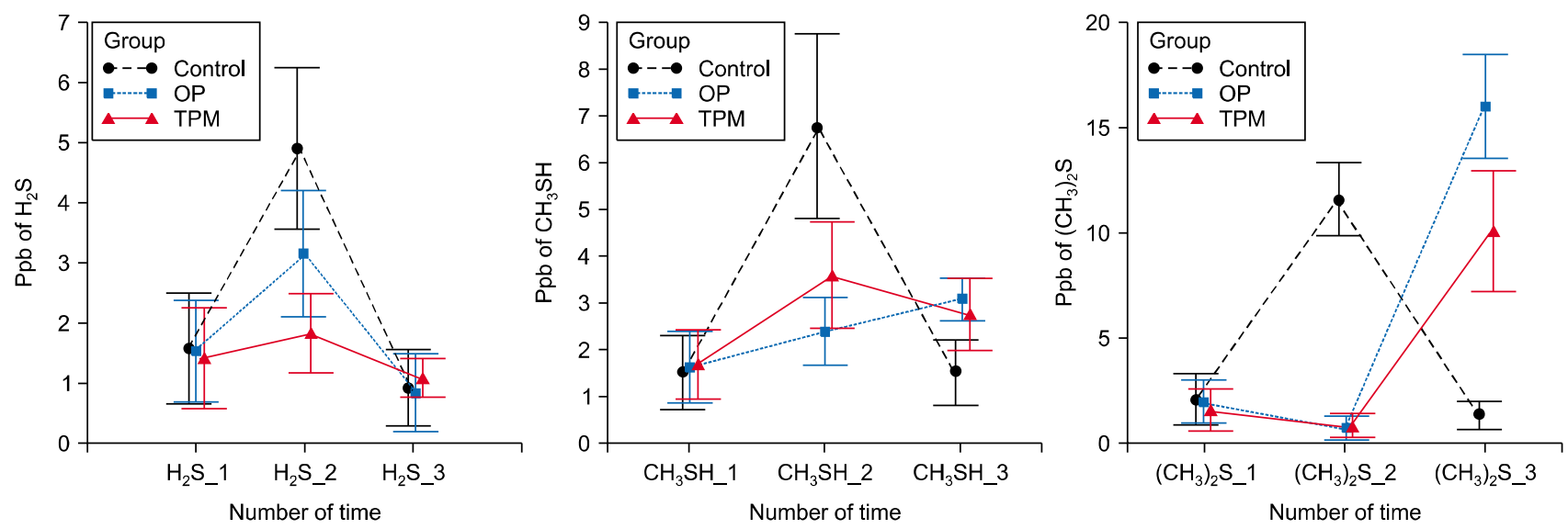

Fig. 3. Halitosis according to groups. OP: oral prophylaxis, TPM: toothpick method.

positive effect on the OP group $(\mathrm{F}=16.353, \mathrm{p}<0.001)$. We also examined changes in subgingival bacterial motility on the smooth surfaces of the teeth. Immediately after treatment, the motility scores were decreased by 32.5 in the general tooth brushing group, and by 50.46 and 56.75 in the OP and TPM groups, respectively, indicating that TPM was the most effective immediately after treatment. When we re-measured subgingival bacterial motility after 5 hours, we observed an increase motility of 32.5 in the general tooth brushing group, 6.62 in the OP group, and 9.83 in the TPM group indicating that OP exerted a more positive effect $(\mathrm{F}=66.575, \mathrm{p}<0.001)$. This is consistent with several studies where the positive effect of TPM on motility subgingival bacterial motility in the biofilm has been reported because the 2-row brush can be readily inserted between the proximal surfaces ${ }^{13,14)}$. Although OP showed a better-sustained effect than TPM in the current study, it was not in accordance with the findings of the study by Park and Han ${ }^{19)}$ in which, TPM was reported to show better continuity than OP. However, this discrepancy may be attributed to the use of natural teeth in the present study, whereas the study by Park and Han ${ }^{19)}$ examined implant prostheses.

Halitosis is highly prevalent in modern society, where social relationships are of value; the importance of halitosis is increasing to an extent that $45 \%$ of Korean adults and $54 \%$ of Korean adolescents seek halitosis treatment $^{20)}$. VSCs are known to be the main causative factors of halitosis, and because they promote the progression to periodontal disease, it is essential to control the growth and activities of the subgingival bacteria that produce $\mathrm{VSCs}^{7)}$. In the current study, we measured the changes in $\mathrm{H}_{2} \mathrm{~S}$, which causes halitosis, immediately after performing the prophylactic treatment and observed an increase of 3.34 in the self-care group (general tooth brushing), 1.61 in the OP group, and 0.41 in the TPM group indicating that TPM was most effective immediately after treatment. After 5 hours, the decreases in the scores for changes in $\mathrm{H}_{2} \mathrm{~S}$ were 4 in the general tooth brushing group, 2.3 in the OP group, and 0.75 in the TPM group indicating that tooth brushing exerted a more positive effect $(F=3.564, p=0.011)$. When we measured changes in $\mathrm{CH}_{3} \mathrm{SH}$, immediately after treatment, there was an increase of 5.25, 0.76, and 1.91 in the general tooth brushing, OP, and TPM groups, respectively; thus, OP was the most effective immediately after treatment. After 5 hours, there was a decrease of $5.25,0.7$, and 0.83 in the general tooth brushing, OP, and TPM groups, respectively, implying that general tooth brushing had a more positive effect $(\mathrm{F}=6.657, \mathrm{p}<0.001)$. When we examined the changes in $\left(\mathrm{CH}_{3}\right)_{2} \mathrm{~S}$ immediately after treatment, there was an increase of 9.58 after general tooth brushing, a decrease of 1.3 after OP, and a decrease of 0.75 after TPM, implying that TPM was the most effective immediately after treatment. After 5 hours, there was a decrease of 10.33 in the general tooth brushing group, an increase of 15.38 in the OP group, and an increase of 9.33 in the TPM group indicating that general tooth brushing had a more 
positive effect $(\mathrm{F}=21.135, \mathrm{p}<0.001)$. Therefore, for all three substances causing halitosis, professional biofilm care was effective immediately after the prophylactic treatment in the current study, which is consistent with the findings of previous studies where tooth brushing and professional cleansing was effective in suppressing halitosis ${ }^{21,22)}$. However, when we analyzed the continuity of the effects against halitosis after 5 hours, self-biofilm care was more effective. This may be attributed to the retention of residual toothpaste in the oral cavity of TPM and OP volunteers when compared to the self-biofilm care method group despite the fact that all three groups used the same paste professional. Among the three components, the levels of $\mathrm{H}_{2} \mathrm{~S}$ and $\mathrm{CH}_{3} \mathrm{SH}$ during the 5 hours measurements demonstrated a similar downward trend in the general tooth brushing group; on the contrary, $\left(\mathrm{CH}_{3}\right)_{2} \mathrm{~S}$ levels were significantly high in this group (Fig. 3).

Both $\mathrm{H}_{2} \mathrm{~S}$ and $\mathrm{CH}_{3} \mathrm{SH}$ levels were lower while $\left(\mathrm{CH}_{3}\right)_{2} \mathrm{~S}$ levels were higher than the halitosis threshold. Therefore, we reasoned that the exhaled air may be composed of gases produced due to food metabolism or certain medications. Moreover, to reiterate, the findings in the study may have been influenced by the presence of the toothpaste in the mouth for longer periods after the self-biofilm care (Table 1$)^{18)}$. Therefore, to precisely compare the effects of each component in causing halitosis, further investigations in these three study groups using an odorless paste are merited. Furthermore, to improve the objectivity of the halitosis assessment, it may be necessary to conduct studies using several types of halitosis-measuring devices.

Our study has several limitations. The amount of time available to verify the continuity of each prophylactic treatment was limited, and the sample size was small. Nevertheless, the highlight of this study is that the effects of self and professional biofilm care on the different tooth surfaces were investigated. This information provides evidence to support the provision of additional specialized oral care by dental hygienists. In future, it will be necessary to perform more in-depth comparative studies, such as analyzing the exact species and abundance of bacteria by culturing after prophylactic treatment or including lingual bacteria to study halitosis more precisely.

\section{Notes}

\section{Conflict of interest}

No potential conflict of interest relevant to this article was reported.

\section{Ethical approval}

This study was exempted from review by the Institutional Review Board at Silla University (IRB No. 1041449-201801-HR-002).

\section{ORCID}

Yu-Rin Kim, https://orcid.org/0000-0001-6440-6745

\section{References}

1. Centers for Disease Control: National health statistics. Retrieved June 26, 2019, from https://knhanes.cdc.go.kr/ knhanes/sub04/sub04_03.do?classType=7 (2015, January 11).

2. Pihlstrom BL, Michalowicz BS, Johnson NW: Periodontal diseases. Lancet 366: 1809-1820, 2005.

https://doi.org/10.1016/S0140-6736(05)67728-8

3. Miyazaki H, Sakao S, Katoh Y, Takehara T: Correlation between volatile sulphur compounds and certain oral health measurements in the general population. J Periodontol 66: 679-684, 1995. https://doi.org/10.1902/jop.1995.66.8.679

4. Doğan B, Asikainen S, Jousimies-Somer H: Evaluation of two commercial kits and arbitrarily primed PCR for identification and differentiation of Actinobacillus actinomycetemcomitans, Haemophilus aphrophilus, and Haemophilus paraphrophilus. J Clin Microbiol 37: 742-747, 1999.

5. Brook I, Walker RI: The relationship between Fusobacterium species and other flora in mixed infection. J Med Microbiol 21: 93-100, 1986. https://doi.org/10.1099/00222615-21-2-93

6. Kishi M, Ohara-Nemoto Y, Takahashi M, et al.: Prediction of periodontopathic bacteria in dental plaque of periodontal healthy subjects by measurement of volatile sulfur compounds in mouth air. Arch Oral Biol 58: 324-330, 2013. https://doi.org/10.1016/j.archoralbio.2012.09.019

7. Scully C, Greenman J: Halitology (breath odour: aetiopathogenesis and management). Oral Dis 18: 333-345, 2012. https://doi.org/10.1111/j.1601-0825.2011.01890.x

8. Yoshida A, Yoshimura M, Ohara N, et al.: Hydrogen sulfide 
production from cysteine and homocysteine by periodontal and oral bacteria. J Periodontol 80: 1845-1851, 2009.

https://doi.org/10.1902/jop.2009.090012

9. Tonzetich J: Production and origin of oral malodor: a review of mechanisms and methods of analysis. J Periodontol 48: 13-20, 1977. https://doi.org/10.1902/jop.1977.48.1.13

10. Bergenholtz A, Hänström L: The plaque-inhibiting effect of hexetidine (Oraldene)-mouthwash compared to that of chlorhexidine. Community Dent Oral Epidemiol 2: 70-74, 1974.

11. Kang BW, Kim GS, Kang HK, et al.: Preventive dentistry. 5th ed. Koonja Publishing Inc, Seoul, pp.130-135, 2014.

12. Oh HM, Song KH: Effect of the tooth pick of the disabled person oral treatment. J Korean Soc Dent Hyg 1: 37-46, 2018.

13. Kang EH, Lim HS, Kim DK, Seong JH: Effectiveness of toothpick method on the reduction of oral malodor. J Korean Acad Oral Health 28: 127-138, 2014.

14. Morita M, Nishi K, Watanabe T: Comparison of 2 toothbrushing methods for efficacy in supragingival plaque removal. The Toothpick method and the Bass method. J Clin Periodontol 25: 829-831, 1998. https://doi.org/10.1111/j.1600-051X.1998.tb02377.x

15. Woo HS, Kim DK: The effect of TBI on PHP index of workers need scaling. J Korean Acad Oral Health 34: 65-71,
2010.

16. Kim SH, Lee HD: An experimental study on the abrasion rate and surface roughness of human dentin occurred by the various methods of dental prophylaxis. J Korean Acad Dent Health 16: 374-391, 1992.

17. Kang HK, Sung MK, Lee MK, Lee BH, Lee JY: Comprehensive periodontics for the dental hygienist. 3rd ed. Soomoonsa Publishing Inc, Seoul, pp.66-68, 2013.

18. Kim HD, Cho YH: Evaluation of oral malodor for patients visiting a dental clinic. J Dent Hyg Sci 9: 461-467, 2009.

19. Park KH, Han GS: The effects of professional tooth cleaning and plaque control instruction on reduction of peri-implantitis. J Dent Hyg Sci 12: 163-170, 2012.

20. Kim YS, Cho JW: Volatile sulfur compound level in Korean measured by use of B\&B checker. Int J Clin Prev Dent 7: 167-177, 2011.

21. Morris PP, Read RR: Halitosis: variations in mouth and total breath odor intensity resulting from prophylaxis and antisepsis. J Dent Res 28: 324-333, 1949. https://doi.org/10.1177/00220345490280031701

22. Tonzetich J, Ng SK: Reduction of malodor by oral cleansing procedures. Oral Surg Oral Med Oral Pathol 42: 172-181, 1976. https://doi.org/10.1016/0030-4220(76)90121-3 